HNO 2016 $\cdot 64: 808-814$

DOI 10.1007/s00106-016-0258-z

Online publiziert: 26. Oktober 2016

(c) Der/die Autor(en) 2016. Dieser Artikel ist

eine Open-Access-Publikation.

CrossMark

J. Encke · J. Kreh · F. Völk · W. Hemmert

Bioanaloge Informationsverarbeitung, Zentralinstitut für Medizintechnik, Technische Universität

München, Garching, Deutschland

\title{
Codierung von Schallsignalen in Aktionspotenziale des auditorischen Nervs
}

und Diskrimination von Sprachsignalen realisiert werden [16]. Darüber hinaus können Modelle auch zur Visualisierung und Erläuterung der Schlüsselfunktionen des Hörvorgangs dienen, was in diesem Beitrag illustriert wird.

\section{Zur Physik des Schalls}

Schall wird durch Schwingungsvorgänge (z. B. einer Lautsprechermembran oder des Vokaltrakts) erzeugt. Im Funktionsbereich des menschlichen Gehörs (etwa $20 \mathrm{~Hz}$ bis $16 \mathrm{kHz}$, [33]) spricht man von Hörschall. In Luft oder anderen elastischen Medien breitet sich Schall mit der jeweiligen Schallgeschwindigkeit (in Luft bei $20^{\circ} \mathrm{C}$ etwa $343 \mathrm{~m} / \mathrm{s}$ [33]) in Form von Wellen aus. Die Wellenlänge $\lambda$ ergibt sich als Quotient der Schallgeschwindigkeit $c$ und der Frequenz $f$ zu $\lambda=c / f$. Die im Hörbereich des Menschen auftretenden Wellenlängen $\lambda$ (in Luft) reichen daher von etwa $2 \mathrm{~cm}$ bei Frequenzen im Bereich um $16 \mathrm{kHz}$ bis zu über $15 \mathrm{~m}$ am unteren Ende des wahrnehmbaren Frequenzbereichs.

\section{Schallverarbeitung im Außen- und Mittelohr}

Die auditorische Informationsverarbeitung beginnt bereits an den beiden Ohrmuscheln, welche zusammen mit Kopf und Oberkörper einfallende Schallwellen richtungsabhängig filtern und so einen wichtigen Beitrag zur Schalllokalisation leisten [2]. Durch die etwa $2 \mathrm{~cm}$ langen Gehörgänge erreichen die Schallwellen das Trommelfell. Hier wird der Luftschall in mechanische Schwingungen umge- setzt, welche über die 3 Gehörknöchelchen Hammer, Amboss und Steigbügel in das mit Lymphflüssigkeit gefüllte Innenohr eingekoppelt werden. Durch die $\lambda / 4$ Resonanz der Gehörgänge und die darauf abgestimmten Eigenschaften der Mittelohrmechanik [25] erreicht das menschliche Gehör im Frequenzbereich um $4 \mathrm{kHz}$ sein Empfindlichkeitsmaximum [33].

\section{Analyse von Schall im Innenohr}

Das Innenohr, wegen seiner Form häufig als (Hör-)Schnecke bezeichnet, besteht aus 3 flüssigkeitsgefüllten Gängen, die sich spiralförmig zur Spitze winden. An der Schneckenbasis befinden sich das ovale und das runde Fenster. Während das runde Fenster nur mit einer dünnen Membran verschlossen ist, bewegt der Steigbügel über das ovale Fenster die Flüssigkeit im Innenohr.

Ein Schnitt durch die Gänge der Schnecke (• Abb. 1) zeigt 2 Membranen, welche den Flüssigkeitsraum in 3 Gänge, meist als Skalen bezeichnet, aufteilen: Die dünne Reissner-Membran trennt die Scala vestibuli von der Scala media. Die wesentlich steifere Basilarmembran mit dem auf ihr befindlichen Corti-Organ trennt die Scala media von der Scala tympani.

Scala tympani und Scala vestibuli sind am oberen Ende der Schnecke durch eine Öffnung, das Helicotrema, verbunden. Die Darstellung vereinfacht sich, wenn die Schnecke gedanklich abgerollt wird (- Abb. 2). Das ovale Fenster, an dem die Fußplatte des Steigbügels ankoppelt, sitzt an der Schneckenbasis in der Scala vestibuli, das runde Fenster in der Scala tym- 


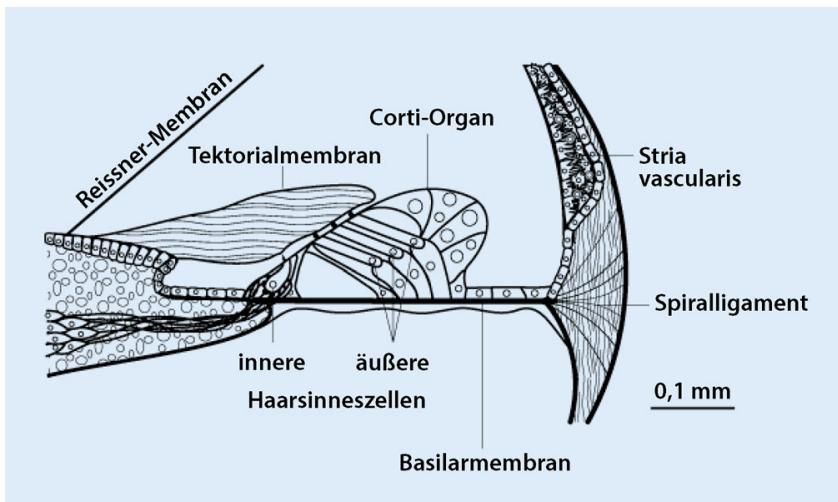

Abb. 1 A Das Corti-Organ. (Mod. nach [13], Copyright beim Autor W. Hemmert)

pani. Da die Reissner-Membran kaum Auswirkungen auf die Flüssigkeitsbewegung im Innenohr hat, kann sie für die folgenden mechanischen Betrachtungen in guter Näherung vernachlässigt werden. Die Trennwand zwischen Scala media und Scala tympani, die aus der Basilarmembran und dem darauf liegenden Corti-Organ besteht, ist im basalen Bereich der Schnecke schmal und steif. Sie wird zur Spitze der Schnecke hin breiter und dünner - und dadurch nachgiebiger. Die positionsabhängige Steifigkeit der Basilarmembran bildet zusammen mit der mitschwingenden Flüssigkeitsmasse ein graduell abgestimmtes Resonanzsystem, das im basalen Bereich bei hohen Frequenzen und an der Spitze bei niedrigen Frequenzen empfindlich ist.

Da die Flüssigkeit im Innenohr näherungsweise als inkompressibel betrachtet werden kann, müssen Bewegungen des Steigbügels, welche die Flüssigkeit in der Scala vestibuli verschieben, durch Auslenkungen des runden Fensters ausgeglichen werden. Bei sehr niedrigen Frequenzen, also bei langsamen Bewegungen, wird die Flüssigkeit entlang der gesamten Scala vestibuli durch das Helicotrema und zurück durch die gesamte Scala tympani verschoben (• Abb. 2a).

\section{》) Bei hohen Frequenzen wird nur wenig Flüssigkeit bewegt}

Bei sehr hohen Frequenzen, also schnellen Bewegungen, setzt die Trägheit der Flüssigkeitsmasse der Bewegung einen nennenswerten Widerstand entgegen.

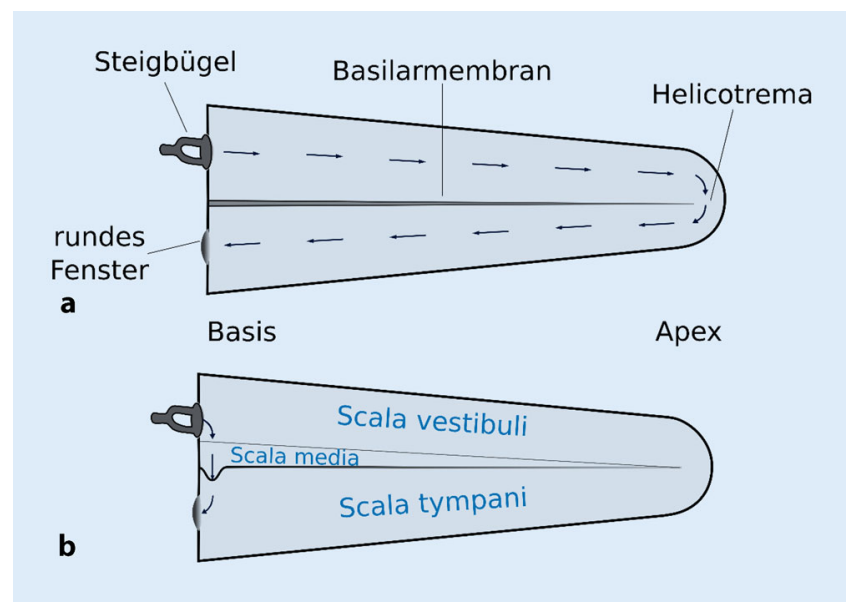

Abb. $2 \Delta$ Schematische Darstellung der Flüssigkeitsverschiebungen im Innenohr bei a niedrigen und $\mathbf{b}$ hohen Frequenzen. (Mod. nach [13], Copyright bei den Autoren J. Kreh und W. Hemmert)

Deshalb wird bei hohen Frequenzen nur wenig Flüssigkeit bewegt: Das von der Steigbügelfußplatte verschobene Volumen gleicht sich mittels Auslenkung der Basilarmembran auf kürzestem Weg, also im basalen Teil der Cochlea, über Scala vestibuli und Scala tympani zum runden Fenster aus (• Abb. 2b).

Bei Frequenzen zwischen den beiden bisher diskutierten hohen und tiefen Extrema kommt es durch die vom Steigbügel eingekoppelten Druckschwankungen sowohl $\mathrm{zu}$ einer direkten Auslenkung der Basilarmembran als auch zu einer Verschiebung der Flüssigkeit in Richtung Helicotrema, also hin zur Spitze der Cochlea, dem Apex. Weil die Steifigkeit der Basilarmembran kontinuierlich abnimmt, wächst dabei deren Auslenkung an und breitet sich in Form einer Wanderwelle in Richtung Helicotrema aus ([1] - Abb. 4a).

Das Auslenkungsmaximum der Wanderwelle entsteht für einen reinen Ton knapp vor der Stelle seiner Resonanzfrequenz auf der Basilarmembran, apikal wird die Welle durch Reibungskräfte im Innenohr schnell gedämpft. So entsteht die charakteristische Form der Wanderwelle: einem relativ flachen Anstieg folgt ein steiler Abfall. Für Töne höherer Frequenz erreicht die Wanderwelle ihr Maximum näher am basalen Anfang der Cochlea, während sie bei tieferen Frequenzen weiter in Richtung Apex läuft (• Abb. 4). Wird über den Steigbügel ein Signal eingekoppelt, das aus mehreren
Tönen verschiedener Frequenz besteht, erreicht die Wanderwelle für jede Frequenz an einer anderen charakteristischen Stelle ihr Schwingungsmaximum, die Spektralkomponenten des Eingangssignals werden entlang der Basilarmembran voneinander getrennt (• Abb. 4).

Durch die Darstellung der Auslenkung zu 2 Zeitpunkten (10 und 10,2 ms nach dem gleichzeitigen Einschalten der Töne) wird die Ausbreitung der Wanderwelle in Richtung Apex sichtbar (- Abb.4). Die maximale Amplitude der aktiven Wanderwelle ist im Vergleich zur passiven etwa 1000-fach größer, zusätzlich ist die Frequenzabstimmung wesentlich schärfer.

\section{》) Das Ruhemembranpotenzial der Sinneszellen im Corti- Organ wird durch Kaliumkanäle reguliert}

Die bisher betrachtete sog. passive Wanderwelle, die bereits 1928 von Georg v. Békésy an präparierten Felsenbeinen eingehend studiert wurde [1], ist jedoch zu breit abgestimmt, um die im Hörsystem beobachtete hohe Frequenzselektivität zu erklären. Für den Hörvorgang im intakten Innenohr spielen die Sinneszellen im Corti-Organ eine entscheidende Rolle. Bei der Beschreibung ihrer elektrischen Funktionalität waren Arbeiten von Prof. Zenner rich- 
tungweisend. So zeigten Gitter, Zenner und Frömter [9], dass das Ruhemembranpotenzial dieser Sinneszellen durch Kaliumkanäle reguliert wird. Kalium $\left(\mathrm{K}^{+}\right)$Ionen diffundieren durch $\mathrm{K}^{+}$Kanäle der Zellmembran in den extrazellulären Raum, und dadurch stellt sich im Zellinneren ein negatives Membranpotenzial ein [9].

Im Corti-Organ sind 2 wichtige Arten von Sinneszellen eingebettet, die inneren und die äußeren Haarsinneszellen (• Abb. 1). Während die Haarbündel der äußeren Haarsinneszellen direkt mit der über dem Corti-Organ liegenden Tektorialmembran verbunden sind, werden die Haarbündel der inneren Haarsinneszellen von Flüssigkeitskräften im engen subtektorialen Spalt ausgelenkt [5, 22].

Die Haarbündel bestehen aus mehreren Reihen von Stereovilli, die mit Querund Tiplinks verbunden sind (• Abb. 3). Wird ein Haarbündel in erregender Richtung ausgelenkt (• Abb. 3, Pfeil), werden die Tiplinks gespannt, und der mit ihnen verbundene Transduktionskanal öffnet sich. Vor allem Kaliumionen $\left(\mathrm{K}^{+}\right)$strömen in die Haarsinneszellen, die durch diesen positiven Strom depolarisiert werden, d.h. ihr Potenzial erhöht sich. Bei Auslenkung der Haarbündel in die andere Richtung schließen sich die Transduktionskanäle, die $\mathrm{K}^{+}$Ionen fließen durch die $\mathrm{K}^{+}$-Kanäle in der Zellmembran wieder $\mathrm{ab}$, und die Zelle wird repolarisiert.

\section{Funktion der äußeren Haarzellen}

Bei denäußeren Haarsinneszellen kommt eine außergewöhnliche Spezialisierung zum Tragen: Elektromotilität. Bei Depolarisation der äußeren Haarsinneszellen verkürzen sie sich, ein Effekt, an dessen Entdeckung und Aufklärung die Arbeitsgruppe um Prof. Zenner maßgeblich beteiligt war [3, 21, 29]. Diese Tübinger Arbeitsgruppe konnte später auch die bei genanntem Vorgang generierte Kraft, die Kontraktion der Zellen und die maximale Frequenz der Abläufe quantifizieren und stellte damit den Weltrekord der schnellsten gemessenen zellulären Motilität auf [6]. Das für die Motilität verantwortliche Protein, wel-

HNO 2016 · 64:808-814 DOI 10.1007/s00106-016-0258-z

(c) Der/die Autor(en) 2016. Dieser Artikel ist eine Open-Access-Publikation.

\title{
J. Encke · J. Kreh · F. Völk · W. Hemmert \\ Codierung von Schallsignalen in Aktionspotenziale des auditorischen Nervs
}

\section{Zusammenfassung}

Die äußeren Haarsinneszellen spielen für die Funktion des Innenohrs eine wesentliche Rolle: Sie verstärken die Schwingung der Basilarmembran bis zu 1000-fach und verschärfen dabei ihre Erregungsmuster. Diese Muster werden von den inneren Haarsinneszellen in Aktionspotenziale des auditorischen Nervs codiert. Bei einer Schädigung der filigranen äußeren Haarsinneszellen, z. B. durch Lärm, kann ein Hörgerät die Amplitude der Erregungsmuster anheben, die fehlende Frequenzselektivität aber nicht wiederherstellen. Zudem zerstört Lärm zunächst Nervenfasern mit niedriger Spontanrate, die für die Codierung von Schall in Störgeräuschen wichtig sind. Dieser Verlust kann mit einer Reintonaudiometrie nicht diagnostiziert werden.

Schlüsselwörter

Hören · Innenohr · Schwerhörigkeit · Auditorische Haarsinneszellen · Computermodelle

\section{Conversion of sound into auditory nerve action potentials}

\begin{abstract}
Outer hair cells play a major role in the hearing process: they amplify the motion of the basilar membrane up to a 1000 -fold and at the same time sharpen the excitation patterns. These patterns are converted by inner hair cells into action potentials of the auditory nerve. Outer hair cells are delicate structures and easily damaged, e. g., by overexposure to noise. Hearing aids can amplify the amplitude of the excitation patterns, but they cannot restore their degraded frequency selectivity.
\end{abstract}

ches dicht gepackt und ausschließlich in der lateralen Zellmembran äußerer Haarsinneszellen vorkommt (• Abb. 3), wurde im Jahr 2000 sequenziert und erhielt aufgrund seiner Schnelligkeit den Namen „Prestin“ [30].

\section{》) Die äußeren Haarsinneszellen bewirken eine bis zu 1000-fache Verstärkung der Wanderwelle}

Zum derzeitigen Stand der Forschung geht man davon aus, dass die äußeren Haarsinneszellen die Auslenkungen der Basilarmembran mit ihren Haarbündeln in ein elektrisches Membranpotenzial transduzieren, welches wiederum eine Kraft in die Schwingung zurückkoppelt [17]. Ähnlich wie auf einer Schaukel, auf der man sich durch die Bewegung der Beine zum richtigen Zeitpunkt „aufschaukelt", bewirken die äußeren Haar-
Noise overexposure also leads to delayed degeneration of auditory nerve fibers, particularly those with low a spontaneous rate, which are important for the coding of sound in noise. However, this loss cannot be diagnosed by pure-tone audiometry.

Keywords Auditory perception $\cdot$ Inner ear $\cdot$ Hearing loss . Cochlear hair cells $\cdot$ Computer models

sinneszellen eine bis zu 1000-fache Verstärkung der Wanderwelle entlang der Basilarmembran, die mithilfe empfindlicher Messungen nachgewiesen wurde $[17,24]$. Neben der Verstärkung der Wanderwellenamplitude bewirkt dieser Prozess auch eine wesentlich schärfer begrenzte Membranauslenkung. Erst dadurch wird die außerordentlich hohe Frequenzselektivität des Gehörs erreicht (• Abb. 4b). Wie der „cochleäre Verstärker“ im Detail funktioniert, ist derzeit noch unverstanden und Gegenstand aktueller Forschung [17]. Zusätzlich zu den genannten Mechanismen kommen Beiträge zu dem aktiven Verstärkungsprozess im Innenohr vom komplexen mechanischen Verhalten der Tektorialmembran $[8,12,14]$ sowie eventuell von einem weiteren „Motor“ im Haarbündel infrage $[4,17]$. 


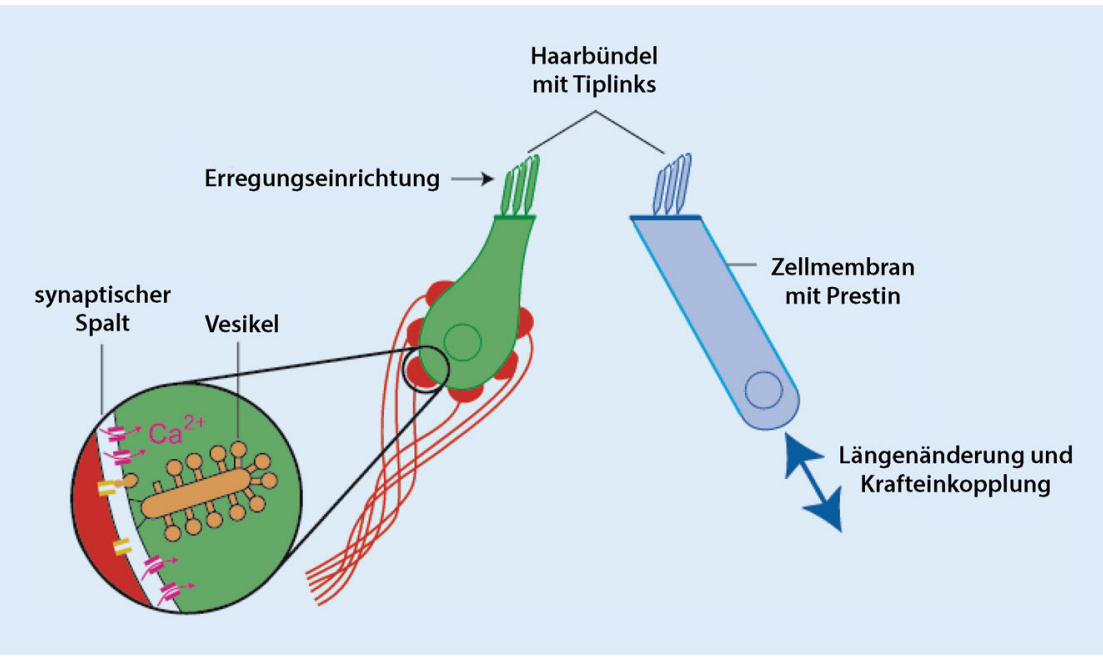

Abb. 3 A Innere und äußere Haarsinneszelle sowie eine vergrößerte afferente Synapse. (Mod. nach [13], Copyright beim Autor W. Hemmert)

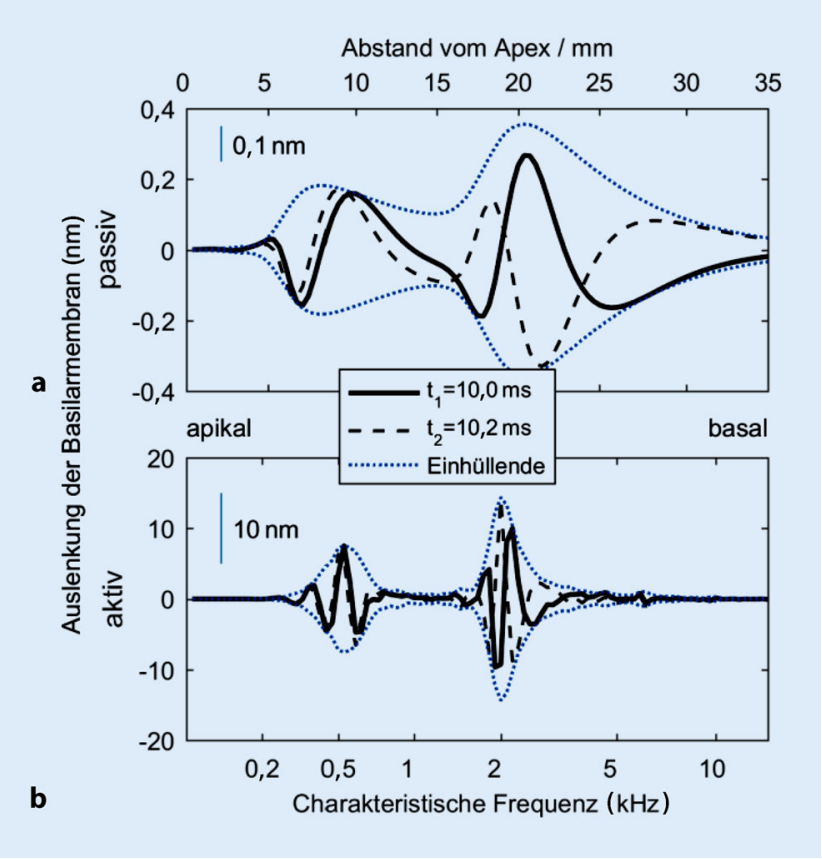

Abb. $4<$ Mit einem Computermodell $[26,27]$ prädizierte Auslenkung der Basilarmembran bei Stimulation mit 2 Tönen $(500 \mathrm{~Hz}$ und $2 \mathrm{kHz}$. a Passives System, b System mit aktiver Verstärkung. Erläuterung s. Text. Hüllkurve der Wanderwelle blau gepunktet. (Mod. nach [13], Copyright bei den Autoren F. Völk und W. Hemmert)

\section{Funktion der inneren Haarsinneszellen}

Die inneren Haarsinneszellen sind die eigentlichen Rezeptorzellen im Innenohr, von ihnen entspringen $95 \%$ der afferenten, also der zum Gehirn führenden Fasern des Hörnervs (• Abb. 3). Die inneren Haarsinneszellen detektieren die (verstärkten und verschärften) mechanischen Schwingungsmuster und codieren sie in Nervenaktionspotenziale. Auch bei den inneren Haarsinneszellen führt Auslenkung der Haarbündel zunächst zu ei-

》) Die inneren Haarsinneszellen
codieren mechanische
Schwingungsmuster in
Nervenaktionspotenziale

In - Abb. 5 ist das entsprechende Rezeptorpotenzial bei Stimulation mit 2 Tönen dargestellt. Das Rezeptorpotenzial ist dabei asymmetrisch, die Depolarisation der

inneren Haarsinneszellen ist größer als die Hyperpolarisation (Erregung durch den $500 \mathrm{~Hz}$ Ton). Bei Frequenzen im Bereich über etwa $1 \mathrm{kHz}$ führt dies zusammen mit der Tiefpasscharakteristik der Zellmembran zum Verlust der Phasenkopplung und zu reiner Depolarisation, die weitgehend der Hüllkurve des Stimulus folgt (2-kHz-Ton).

Die Depolarisation einer inneren Haarsinneszelle führt zur Öffnung von spannungsgesteuerten $\operatorname{Kalzium}\left(\mathrm{Ca}^{2+}\right)$ Kanälen (• Abb. 3), die an den aktiven Zonen der Synapsen lokalisiert sind [11]. Die einströmenden $\mathrm{Ca}^{2+}$-Ionen bewirken eine Fusion der synaptischen Vesikel mit der äußeren Zellmembran. Da die Geschwindigkeit der Repolarisation der inneren Haarsinneszellen begrenzt ist, kann das Rezeptorpotenzial der periodischen Auslenkung der Haarbündel nur bei niedrigen Stimulusfrequenzen folgen (• Abb. 5, 500-Hz-Ton). Bei Frequenzen ab etwa $1 \mathrm{kHz}$ [23] reagieren die inneren Haarsinneszellen nur noch mit reiner Depolarisation, die der Hüllkurve des Schallsignals folgt (• Abb. 5, 2-kHzTon).

\section{Codierung von Schall auf dem auditorischen Nerv}

Durch die Fusion der synaptischen Vesikel wird der darin enthaltene Neurotransmitter (Glutamat) in den synaptischen Spalt ausgeschüttet [10]. Das Glutamat diffundiert über den Spalt und bindet an die Rezeptoren der Postsynapse, wodurch ein Aktionspotenzial ausgelöst wird, das in Richtung Gehirn weiterläuft. Bei Schall hohen Pegels depolarisieren die inneren Haarsinneszellen stärker, dadurch wird mehr Neurotransmitter ausgeschüttet, und die Nervenfasern "feuern“ schneller, maximal 200- bis 300-mal pro Sekunde [28].

\section{》) Bei Schall hohen Pegels depolarisieren die inneren Haarsinneszellen stärker}

Zur Codierung des großen Dynamikbereichs des hörbaren Schalls zwischen Hör- und Schmerzschwelle (etwa $120 \mathrm{~dB}$, d.h. ein Unterschied um den Faktor 


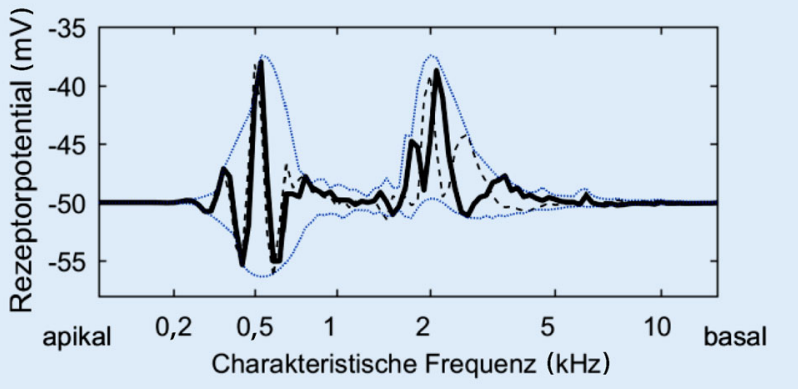

Abb. 5 ॥ Mit einem Computermodell [26, 27] prädiziertes Rezeptorpotenzial der inneren Haarsinneszellen entlang der Cochlea bei Stimulation mit 2 Tönen $(500 \mathrm{~Hz}$ und $2 \mathrm{kHz}$ ) zu 2 Zeitpunkten ( 10 und 10,2 ms nach dem gleichzeitigen Einschalten der Töne). Erläuterung s. Text. (Mod. nach [13], Copyright bei den Autoren F. Völk und W. Hemmert)

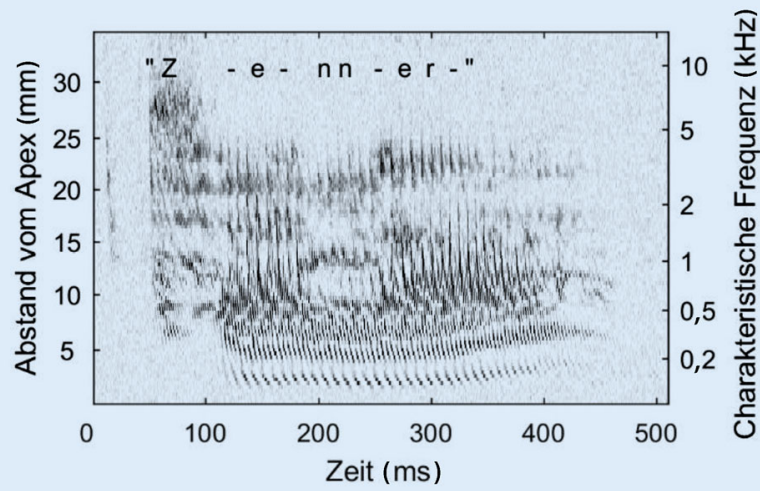

Abb. 6 ॥ Mittels eines Computermodells [26, 27] prädizierte Aktivität der Fasern des auditorischen Nervs mit hoher Spontanrate in Reaktion auf das gesprochene Wort „Zenner". (Mod. nach [13], Copyright beim Autor)
1:1 Mio.!) tragen Fasern mit verschiedenen Empfindlichkeiten und damit zusammenhängend mit verschiedenen Spontanraten bei. Fasern mit hoher Spontanrate „feuern“ bereits ohne Schall, bedingt durch das thermische Rauschen („spontan“). Sie sind die empfindlichsten Fasern und bestimmen die klinisch im Tonaudiogramm gemessene Schwelle. Die Feuerrate dieser Fasern steigt bei einer Pegelerhöhung schnell an, sie erreichen bereits ab etwa $20 \mathrm{~dB}$ oberhalb ihrer Schwelle ihre maximale Feuerrate. Dadurch können diese Fasern nur einen geringen Dynamikbereich abdecken. Fasern mit mittlerer und niedriger Spontanrate haben jeweils höhere Schwellen und langsamer mit dem Pegel steigende Feuerraten, die kaum in Sättigung gelangen [28]. Es wird deshalb vermutet, dass die beiden letztgenannten Gruppen von Fasern bei der Codierung von Sprache in Störgeräuschen eine wesentliche Rolle spielen [16, 18].

Da die Wanderwelle die im Eingangssignal enthaltenen Spektralkomponenten aufspaltet, wird die in Schallen enthaltene Information frequenzspezifisch an das Gehirn geleitet. Wenn also eine bestimmte Gruppe von Nervenfasern aktiv ist, „weiß“ das Gehirn anhand der Stelle entlang der Basilarmembran, an der die Synapsen dieser Fasern entspringen, aus welchen Spektralkomponenten das Schallsignal besteht.

Dieses Prinzip wird Tonotopie genannt und bleibt als Orts-FrequenzKartierung entlang des auditorischen
Pfads bis in den Kortex erhalten. Die Intensität eines Schalls wird als Kombination der Feuerraten der 3 genannten Gruppen von Nervenfasern codiert. Die Summe der aktiven Hörnervenfasern liefert dem Gehirn somit ein Bild, welches ähnlich einem Spektrogramm visualisiert werden kann.

\section{》) Die Tonotopie bleibt entlang des auditorischen Pfads bis in den Kortex erhalten}

Ein solches Bild kann mit aktuellen Modellen berechnet werden und ist für das Beispiel des gesprochenen Worts "Zenner" in - Abb. 6 dargestellt. Jeder graue Punkt repräsentiert ein einzelnes Aktionspotenzial. Treten viele Aktionspotenziale gleichzeitig auf, entstehen dunkle Bereiche. Bei tiefen Frequenzen können die Frequenzkomponenten des Schallsignals sowohl anhand ihrer Position entlang der Cochlea abgelesen werden (Tonotopie), als auch - bedingt durch die Phasenkopplung - im Zeitverlauf. In - Abb. 6 kann so die Grundfrequenz und die Frequenz der ersten Harmonischen durch Zählen der Erregungsmaxima pro Zeiteinheit bestimmt werden. Bei Frequenzen oberhalb von etwa $3 \mathrm{kHz}$ geht die Phasenkopplung vollständig verloren. Es wird jedoch immer noch die Hüllkurve des Sprachsignals mit hoher zeitlicher Präzision codiert (in • Abb. 6 sichtbar im Bereich zwischen 1 und $6 \mathrm{kHz}$ ).
Aus Abb. 6 geht ebenfalls hervor, dass bei tiefen Frequenzen einzelne Spektralkomponenten aufgelöst werden, dagegen wird der Hörnerv bei höheren Frequenzen bei globalen Energiemaxima des Signals erregt, wodurch die für die $\mathrm{Re}$ präsentation von Sprachsignalen wichtigen Formanten codiert werden. Durch die Analyse des zeitlichen Verlaufs der Sprachformanten können die gesprochenen Phoneme identifiziert und Sprachsignale auch in Störgeräuschen verstanden werden.

\section{Konsequenzen von Hörschäden}

Bei Schädigung der äußeren Haarsinneszellen, z. B. durch Lärm, wird zunächst der aktive Verstärkungsprozess beeinträchtigt. Die Amplitude der Wanderwelle und damit die Empfindlichkeit des Gehörs nimmt ab, was sich klinisch im Tonaudiogramm manifestieren würde. Eine reine Schwellenerhöhung könnte durch ein Hörgerät mit entsprechender Verstärkung kompensiert werden. Der Vergleich der für Normalhörende realistischen aktiven Wanderwelle (• Abb. 4b) mit der Wanderwelle ohne Verstärkung durch die äußeren Haarsinneszellen (- Abb. 4a) zeigt jedoch, dass neben der Verringerung der Amplitude auch die Breite der Abstimmkurve stark zunimmt, d. h. die Frequenzselektivität der Wanderwelle und damit der neuronalen Erregungsmuster nimmt ab.

Die hohe Frequenzselektivität des intakten Innenohrs lässt sich durch externe 
Verstärkung nicht wiederherstellen, da diese nur die Amplitude der in • Abb. 4a gezeigten Wanderwelle vergrößert. Dies deckt sich mit der Erfahrung, dass $\mathrm{Pa}$ tienten mit Hörgeräteversorgung Sprache zwar hören, Konversationen in störgeräuschbehafteten Umgebungen jedoch nur schwer folgen können.

\section{》) Die Frequenzselektivität des Innenohrs lässt sich durch externe Verstärkung nicht wiederherstellen}

Ein weiterer Schädigungsmechanismus des Gehörs bei zu hoher und/oder langer Schallbelastung wurde kürzlich entdeckt: Durch die akkumulierte Ausschüttung von Neurotransmitter kann dieser toxisch wirken. Dies schädigt Nervenfasern, jedoch weniger die Fasern mit hoher Spontanrate, die Töne geringen Pegels codieren, sondern insbesondere Fasern mit niedriger Spontanrate. Letztere werden für die Codierung von Schall höherer Amplitude benötigt, und ihr Fehlen bleibt in klinischen Hörschwellenmessungen unentdeckt [18]. Da die Degeneration der Nervenfasern zudem mit einer Verzögerung von Monaten bis Jahren einsetzt [7], wurde dieser Effekt lange nicht erkannt. Die Degeneration führt vermutlich zur Beeinträchtigung des Sprachverstehens in Störgeräuschen $[18,16]$ und kann durch Hörgeräte mit reiner Verstärkung nicht ausgeglichen werden. Zur Wiederherstellung bzw. der Verbesserung der Kommunikationsfähigkeit ist deshalb die Entwicklung neuer Methoden zur aktiven Unterdrückung von Störgeräuschen essenziell. Erste Ansätze dazu haben bereits Einzug in die Technologie moderner Hörgeräte gefunden, weitere Entwicklungen sind wünschenswert.

\section{Fazit für die Praxis}

- Innenohrmodelle generieren realistische Aktionspotenzialfolgen des gesamten auditorischen Nervs für beliebige Schallsignale.

- Äußere Haarsinneszellen im Innenohr verstärken die Schwingung der
Basilarmembran bis zu 1000-fach und verschärfen dabei die Erregungsmuster.

- Innere Haarsinneszellen codieren die verstärkten und verschärften Erregungsmuster in Aktionspotenziale des auditorischen Nervs.

- Bei tiefen Frequenzen erscheinen die Aktionspotenziale des Hörnervs synchron zur Stimulusfrequenz (Phasenkopplung).

- Diese Phasenkopplung wird ab etwa 1 kHz schwächer, bei höheren Frequenzen folgen die Aktionspotenziale der Hüllkurve des Stimulus.

- Bei einer Schädigung der äußeren Haarsinneszellen, z. B. durch Lärm, kann nur die Amplitude der Erregungsmuster durch ein Hörgerät angehoben werden, die fehlende Frequenzselektivität kann nicht mehr rekonstruiert werden.

- Lärm schädigt auch die Nervenfasern mit niedriger Spontanrate, welche für die Codierung von Schall in Umgebungen mit Störgeräuschen wichtig sind. Dieser Verlust bleibt im klinischen Audiogramm unentdeckt.

\section{Korrespondenzadresse}

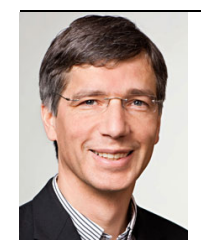

Prof. Dr.-Ing. W. Hemmert Bioanaloge Informationsverarbeitung, Zentralinstitut für Medizintechnik, Technische Universität München Boltzmannstr. 11, 85748 Garching, Deutschland werner.hemmert@tum.de

Danksagung. Diese Arbeit wurde von der Deutschen Forschungsgemeinschaft (DFG) im Rahmen des Schwerpunktprogramms SPP 1608 „Ultrafast and temporally precise information processing: normal and dysfunctional hearing" (HE6713/1-1 and 1-2) sowie vom Bundesministerium für Bildung und Forschung im Rahmen des Münchner Bernsteinzentrums für Computational Neuroscience (01GQ1004B) gefördert.

\section{Einhaltung ethischer Richtlinien}

Interessenkonflikt. J. Encke, J. Kreh, F. Völk und W. Hemmert geben an, dass kein Interessenkonflikt besteht.

Dieser Beitrag beinhaltet keine von den Autoren durchgeführten Studien an Menschen oder Tieren.
Open Access. Dieser Artikel wird unter der Creative Commons Namensnennung 4.0 International Lizenz (http://creativecommons.org/licenses/by/4.0/deed. de) veröffentlicht, welche die Nutzung, Vervielfältigung, Bearbeitung, Verbreitung und Wiedergabe in jeglichem Medium und Format erlaubt, sofern Sie den/die ursprünglichen Autor(en) und die Quelle ordnungsgemäßnennen, einen Linkzur Creative Commons Lizenz beifügen und angeben, ob Änderungen vorgenommen wurden.

\section{Literatur}

1. Békésy G (1928) Zur Theorie des Hörens. Phys Z 29:793-810

2. Blauert J (1974) Räumliches Hören. S. Hirzel, Stuttgart

3. Brownell WE, Bader CR, Bertrand D, Ribaupierre Y (1985) Evoked mechanical responses of isolated cochlear outer hair cells. Science 227(4683):194-196

4. Chan DK, Hudspeth AJ (2005) Ca2+ currentdriven nonlinear amplification by the mammalian cochlea in vitro. Nat Neurosci 8(2):149-155

5. Freeman DM, Weiss TF (1988) The role of fluid inertia in mechanical stimulation of hair cells. Hear Res 35(2-3):201-207

6. Frank G, Hemmert W, Gummer AW (1999) Limiting dynamics of high-frequency electromechanical transduction of outer hair cells. Proc Natl Acad Sci 96(8):4420-4425

7. Furman AC, Kujawa SG, Liberman MC (2013) Noise-induced cochlear neuropathy is selective for fibers with low spontaneous rates. J Neurophysiol 110(3):577-586

8. Ghaffari R, Aranyosi AJ, Freeman DM (2007) Longitudinally propagating traveling waves of the mammalian tectorial membrane. Proc Natl Acad SciUSA 104(42):16510-16515

9. Gitter A, Zenner H, Frömter E (1986) Membrane potential and ion channels in isolated outer hair cells of Guinea pig cochlea. ORL 48(2):68-75

10. Glowatzki E, Fuchs PA (2002) Transmitter release at the hair cell ribbon synapse. Nat Neurosci 5(2):147-154

11. Graydon CW, Cho S, Li G-L, Kachar B, Gersdorff $H$ (2011) Sharp Ca2+ nanodomains beneath the ribbon promote highly synchronous multivesicular release at hair cell synapses. J Neurosci 31(46):16637-16650

12. Gummer AW, Hemmert W, Zenner HP (1996) Resonant tectorial membrane motion in the inner ear: its crucial role in frequency tuning. Proc Natl Acad Sci USA 93(16):8727-8732

13. Hemmert W (kein Datum) How our Ear Works. http://www.imetum.tum.de/en/researchgroups/bai/research/how-our-ear-works/. Zugegriffen: 8.8.2016

14. Hemmert W, Zenner H-P, Gummer AW (2000) Three-dimensional motion of the organ of corti. Biophys J 78(5):2285-2297

15. Holmberg M, Hemmert W (2004) An auditory model for coding speech into nerve-action potentials. Proc Joint Congr CFA DAGA 4:773-774

16. Holmberg M, Gelbart D, Hemmert W (2007) Speech encoding in a model of peripheral auditory processing. Speech Commun 49(12):917-932

17. Ashmore J, Avan P, Brownell WE, Dallos P, Dierkes K, Fettiplace R et al (2010) The remarkable cochlear amplifier. Hear Res 266(1-2):1-17

18. Kujawa SG, Liberman MC (2009) Adding insult to injury: cochlear nerve degeneration after 
„temporary" noise-induced hearing loss. JNeurosci 29(45):14077-14085

19. Meddis R(kein Datum) Matlab model of the auditory periphery (MAP). http://www.essexpsychology. macmate.me/HearingLab/modelling.html. Zugegriffen: 22.3 .2014

20. Meddis R, Lopez-Poveda EA (2010) Auditory periphery:from pinna to auditory nerve. In:Meddis R, Fay RR, Lopez-Poveda EA, Popper AN (Hrsg) Computational models of the auditory system, $\mathrm{Bd}$. 2.Springer, Boston, S7-38

21. Oliver D, He DZ, Klocker N, Ludwig J, Schulte U, Waldegger Set al (2001) Intracellular anions as the voltage sensor of prestin, the outer hair cell motor protein. Science 292(5525):2340-2343

22. Sellick PM, Russell IJ (1980) The responses of inner hair cells to basilar membrane velocity during low frequency auditory stimulation in the guinea pig cochlea. Hear Res 2(3-4):439-445

23. Palmer AR, Russell IJ (1986) Phase-locking in the cochlear nerve of the guinea-pig and its relation to the receptor potential of inner hair-cells. Hear Res 24(1):1-15

24. Rhode WS (1971) Observations of the vibration of the basilar membrane in squirrel monkeys using the Mössbauer technique. J Acoust Soc Am 49(4B):1218-1231

25. Jorge RJ, Zenner $\mathrm{H}$, Hemmert W, Burkardt C, Gummer A (1997) Laservibrometrie: Ein Mittelohr-und Kochleaanalysator zur nicht-invasiven Untersuchung von Mittel-und Innenohrfunktionsstörungen. HNO 45(12):997-1007

26. Rudnicki M, Hemmert W (2016) Cochlea: inner ear models in Python. https://github. com/mrkrd/cochlea, http://dx.doi.org/10.5281/ zenodo.61462.Zugegriffen:25.7.2016

27. Rudnicki M, Schoppe O, Isik M, Völk F, Hemmert W (2015) Modeling auditory coding: from sound to spikes. Cell Tissue Res 361(1):159-175

28. Yates GK, Winter IM, Robertson D (1990) Basilar membrane nonlinearity determines auditory nerve rate-intensity functions and cochlear dynamic range. Hear Res 45(3):203-219

29. Zenner HP (1986) Motile responses in outer hair cells. Hear Res 22(1):83-90

30. Zheng J, Shen W, He DZ, Long KB, Madison LD, Dallos $\mathrm{P}$ (2000) Prestin is the motor protein of cochlear outer hair cells. Nature 405(6783):149-155

31. Zilany MS, Bruce IC, Nelson PC, Carney LH (2009) A phenomenological model of the synapse between the inner hair cell and auditory nerve: long-term adaptation with power-law dynamics. J Acoust Soc Am 126(5):2390-2412

32. Zilany M, Bruce I, Carney L (2014) Updated parameters and expanded simulation options for a model of the auditory periphery. J Acoust Soc Am 135(1):283-286

33. Zollner M, Zwicker E (1993) Elektroakustik. Springer, Heidelberg

\section{Die meisten Schlaganfälle sind vermeidbar}

Dass die meisten Schlaganfälle vermeidbar sind, hatte vor sechs Jahren bereits die erste Phase der Interstroke-Studie gezeigt (und die Global Burden of Disease Study kam 2013 zu ähnlichen Ergebnissen). Die InterstrokeStudie basierte damals auf dem Vergleich von 3.000 Schlaganfallpatienten aus 22 Ländern mit einer gleich großen Zahl von Gesunden oder Patienten mit anderen Krankheiten. Für die neue Publikation konnte das Team vom Population Health Research Institute an der McMaster Universität in Hamilton insgesamt 13.447 Schlaganfallpatienten (davon 10.388 mit ischämischem Schlaganfall und 3.059 mit einer intrazerebralen Blutung) und 13.472 Kontrollen aus 32 Ländern rekrutieren. Als der mit Abstand wichtigste Risikofaktor stellte sich arterielle Hypertonie heraus, mit einem dreifach erhöhten Schlaganfallrisiko (Odds Ratio 2,98; 95\%-Konfidenzintervall 2,72-3,28). Da eine arterielle Hypertonie in den meisten Gesellschaften stark verbreitet ist, beträgt das attributable Risiko für die Bevölkerung (PAR) 47,9\%. Mit anderen Worten: Fast die Hälfte aller Schlaganfälle ist die Folge eines über Jahre zu hohen Blutdrucks. An zweiter Stelle steht bereits der Bewegungsmangel. Wer vier Stunden in der Woche Sport treibt oder anderweitig körperlich aktiv ist, kann sein Schlaganfallrisiko um $40 \%$ senken $(\mathrm{OR} 0,60(0,52-0,70)$, PAR $35,8 \%)$. In den westlichen Ländern sind Übergewicht und Adipositas ein Public-Health-Problem. Ein Taille-Hüfte-Verhältnis im oberen Drittel ist hier für 36,7\% aller Schlaganfälle verantwortlich, in Ost- und Zentraleuropa beträgt das PAR nur 2,8\%. Das globale PAR liegt bei $18,6 \%$ (OR 1,44). Das individuelle Risiko ist demnach weitaus geringer als bei einer arteriellen $\mathrm{Hy}$ pertonie oder bei Bewegungsmangel. Eine ungesunde Ernährung leistet ebenfalls einen wichtigen Beitrag: Menschen im oberen Drittel des modifizierten „Alternative Healthy Eating Index", einer vom US-Landwirtschaftsministerium entworfenen Bewertung, hatten ein um $40 \%$ vermindertes Schlaganfall-Risiko (OR 0,60; 0,53-0,67). In westlichen Ländern ist ungesunde Ernährung für 33,3\% der Schlaganfälle verantwortlich.

Der wichtigste individuelle Risikofaktor sind kardiale Erkrankungen, sprich Vorhofflimmern oder -flattern (OR 3,17 (2,68-3,75). Auch hohe Cholesterinwerte stellen ein Ri- siko dar (OR 1,84; 1,65-2,06), ebenso psychosoziale Faktoren (OR 2,20; 1,78-2,72) und Diabetes mellitus (OR 1,16; 1,05-1,30). Last but not least hilft der Verzicht auf das Rauchen (OR 1,67; 1,49-1,87, PAR 12,4\%) und auf übermäßigen Alkoholkonsum (OR 2,09; 1,642,67, PAR 5,8\%) dabei, einen Schlaganfall zu vermeiden.

\section{Quelle: Deutsches Ärzteblatt}

www.aerzteblatt.de

basierend auf: Lancet (2016) doi: 10.1016/ S0140-6736(16)30506-2 